\title{
Nonuniform Illumination Correction Algorithm for Underwater Images Using Maximum Likelihood Estimation Method
}

\author{
Sonali Sachin Sankpal ${ }^{1}$ and Shraddha Sunil Deshpande ${ }^{2}$ \\ ${ }^{1}$ E \& TC Engineering Department, P.V.P.I.T., Sangli-Vita Road, Budhgaon, Sangli, Maharashtra 416304, India \\ ${ }^{2}$ Electronics Engineering Department, Walchand College of Engineering, Sangli Miraj Road, Vishrambaug, Sangli, \\ Maharashtra 416415, India \\ Correspondence should be addressed to Sonali Sachin Sankpal; sssankpal.etc@pvpitsangli.edu.in
}

Received 28 September 2015; Revised 30 December 2015; Accepted 31 December 2015

Academic Editor: Mohamed Ichchou

Copyright (C) 2016 S. S. Sankpal and S. S. Deshpande. This is an open access article distributed under the Creative Commons Attribution License, which permits unrestricted use, distribution, and reproduction in any medium, provided the original work is properly cited.

\begin{abstract}
Scattering and absorption of light is main reason for limited visibility in water. The suspended particles and dissolved chemical compounds in water are also responsible for scattering and absorption of light in water. The limited visibility in water results in degradation of underwater images. The visibility can be increased by using artificial light source in underwater imaging system. But the artificial light illuminates the scene in a nonuniform fashion. It produces bright spot at the center with the dark region at surroundings. In some cases imaging system itself creates dark region in the image by producing shadow on the objects. The problem of nonuniform illumination is neglected by the researchers in most of the image enhancement techniques of underwater images. Also very few methods are discussed showing the results on color images. This paper suggests a method for nonuniform illumination correction for underwater images. The method assumes that natural underwater images are Rayleigh distributed. This paper used maximum likelihood estimation of scale parameter to map distribution of image to Rayleigh distribution. The method is compared with traditional methods for nonuniform illumination correction using no-reference image quality metrics like average luminance, average information entropy, normalized neighborhood function, average contrast, and comprehensive assessment function.
\end{abstract}

\section{Introduction}

When light travels through the water it deviates from its path which is called scattering of the light. The scattering is caused by water molecules themselves, dissolved chemical compounds in water, and suspended particles. This scattering decreases visibility in water. As you go deeper in the water the scattering of natural light increases which results in poor visibility in deep water. This degrades the performance of the underwater imaging system and it goes worse for deep water imaging.

Propagation of light in water is discussed by Jaffe in [1]. His image formation model divides reflected light in three components given by the following:

$$
E_{T}=E_{d}+E_{f}+E_{b} \text {, }
$$

where $E_{T}$ is total irradiance, $E_{d}$ is direct component, $E_{f}$ is forward scattered component, and $E_{b}$ is backscattered component.

Forward and backward scattering of light degrade the quality of underwater images. The degradation includes limited contrast, addition of blur, and diminished colors.

The image enhancement techniques are used to compensate for the degradation like contrast enhancement and color correction. Underwater visibility can be increased by using artificial light source. But artificial light adds nonuniform illumination to image. This problem is ignored by many researchers. Very few researchers proposed a correction method for nonuniform illumination in underwater images.

The nonuniform illumination problem in underwater images is shown in Figure 1. An artificial light source is used in underwater imaging system to increase visibility in water. The light rays from artificial light source are reflected 


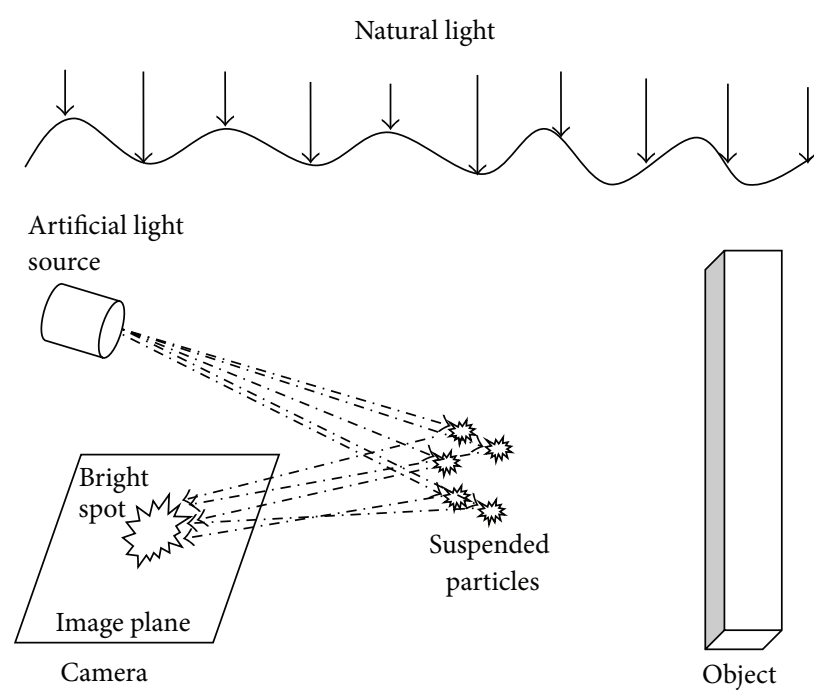

FIGURE 1: Nonuniform illumination created in underwater image.

in backward direction without reaching the objects in the scene. This is called backward scattering. This reflected light produces bright spot in the center and is surrounded by dark region [2]. This illuminates the scene in nonuniform fashion. It is assumed that pixel intensity values of underwater images are dominated by Rayleigh scattering [3]. The scattering of light is wavelength dependent so for correction of nonuniform illumination each color component (R, G, B) should be processed separately. While providing solution for nonuniform illumination, the method assumes that the underwater images are Rayleigh distributed [4-6]. Consider

$$
f_{x}(x)= \begin{cases}\frac{x}{\alpha} e^{-x^{2} / 2 \alpha^{2}}, & x \geq 0 \\ 0, & \text { otherwise. }\end{cases}
$$

The Rayleigh distribution function is bell shaped with major amount of pixels being concentrated in middle intensity levels. The probability distribution function of Rayleigh distributed random variable $x$ with parameter $\alpha$ is given by (2), where $\alpha$ is mean square value of $x$.

The method maps image to Rayleigh distribution by estimation of parameters using maximum likelihood method. The principle of maximum likelihood method [7] says that given observations $x_{1}, x_{2}, \ldots, x_{n}, f_{x}\left(x_{1}, x_{2}, \ldots, x_{n} ; \theta\right)$ are function of $\theta$ alone, and the value of $\theta$ that maximizes the above probability density function is the most likely value for $\theta$, and it is chosen as its maximum likelihood estimation $\theta_{\mathrm{ML}}$.

This paper proposed a method for nonuniform illumination correction for underwater images. The rest of the paper is organized as follows. Section 2 discussed state of the art for the problem, in Section 3 a proposed method for nonuniform illumination correction is explained, the image quality metrics are discussed in Section 4, results are reported in Section 5, and conclusion is given in Section 6.

\section{Literature Review}

Arnold-Bos et al. [8] suggested that global histogram equalization is not suitable when illumination in image is unequal, and local methods are needed. They proposed a method of histogram clipping and then equalized contrast by division method.

Bazeille et al. [9] suggested homomorphic filtering to correct nonuniform illumination. The maximum and minimum coefficient values selected by them are $\gamma_{H}=2.5$ and $\gamma_{L}=0.5$.

Garcia et al. [10] compared four methods for nonuniform illumination correction. The first method uses illumination reflectance model and illumination correction is obtained as

$$
\widetilde{r}(x, y)=\frac{f(x, y)}{f_{s}(x, y)} \delta,
$$

where $f(x, y)$ is original and $f_{s}(x, y)$ is smoothed version of image. $\delta$ is constant. Contrast Limited Adaptive Histogram Equalization (CLAHE) and homomorphic filter are second and third methods. In fourth method illumination field is subtracted from original image for nonuniform illumination correction.

Borgetto et al. [11] also discussed two methods for illumination correction: one method used homomorphic filter and the other is based on CCD camera radiometric correction required for mosaicking.

Prabhakar and Praveen Kumar [12] proposed algorithm for enhancement of underwater images. They used homomorphic filter to correct nonuniform illumination.

\section{Proposed Method}

The proposed method in this paper improves quality of underwater images by correcting nonuniform illumination in image. Like traditional methods proposed by Iqbal et al. in $[13,14]$, histogram of the image is modified for correction. The method in this paper applied histogram stretching. The detailed process is given in Figure 2. 


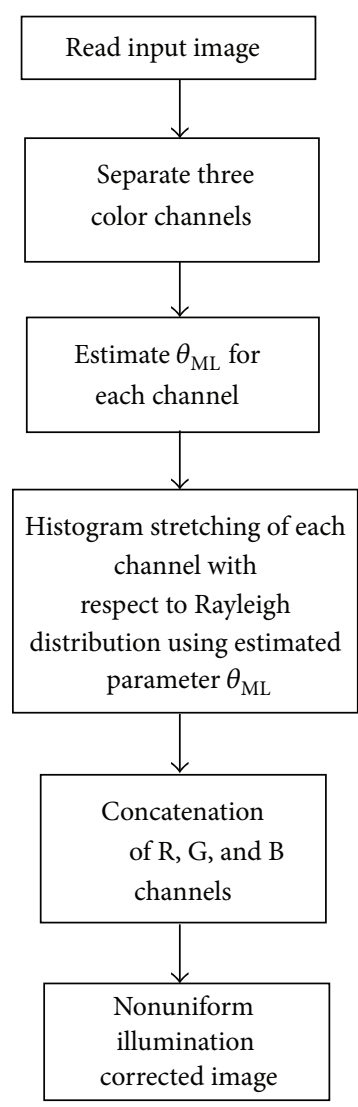

FIGURE 2: Process of proposed algorithm.

Input image is first decomposed into three channels (red, green, and blue). Then histogram stretching is performed on individual channels. With the assumption that each of $\mathrm{R}, \mathrm{G}$, and B channel is Rayleigh distributed, histogram stretching is done with respect to Rayleigh distribution. The scale parameter is estimated from given image. So the histogram stretching is adaptive. The scale parameter $(\theta)$ is estimated using the maximum likelihood method given below. First find log likelihood function of image as given by

$$
l(\theta)=\ln \prod_{i=1}^{n} f\left(x_{i} ; \theta\right) .
$$

Here $f\left(x_{i} ; \theta\right)$ is a probability density function of $x_{i}$ with scale parameter $\theta$. Consider

$$
\begin{aligned}
& l(\theta)=\ln \prod_{i=1}^{n} \frac{x_{i}}{\theta} e^{-x_{i}^{2} / 2 \theta}, \\
& l(\theta)=\sum_{i=1}^{n} \ln \frac{x_{i}}{\theta} e^{-x_{i}^{2} / 2 \theta}, \\
& l(\theta)=\sum_{i=1}^{n} \ln x_{i}-n \ln \theta-\sum_{i=1}^{n} \frac{x_{i}^{2}}{2 \theta} .
\end{aligned}
$$

Then find the value of $\theta$ where $\log$ likelihood function has maximum value. It can be determined by taking a derivative of $\log$ likelihood function with respect to $\theta$ and equate it to zero:

$$
\frac{\partial}{\partial \theta} l(\theta)=0
$$

Solve (6) for $\theta$ :

$$
\begin{aligned}
\frac{\partial}{\partial \theta}\left\{\sum_{i=1}^{n} \ln x_{i}-n \ln \theta-\sum_{i=1}^{n} \frac{x_{i}^{2}}{2 \theta}\right\} & =0, \\
-\frac{n}{\theta}-\sum_{i=1}^{n} \frac{x_{i}^{2}}{2}\left(-\frac{1}{\theta^{2}}\right) & =0, \\
\sum_{i=1}^{n} \frac{x_{i}^{2}}{2 \theta^{2}} & =\frac{n}{\theta}, \\
\theta_{\mathrm{ML}} & =\frac{1}{2 n} \sum_{i=1}^{n} x_{i}^{2} .
\end{aligned}
$$

Obtained $\theta$ in (10) is estimated maximum likelihood value $\theta_{\mathrm{ML}}$. Thus maximum likelihood values are estimated for all three channels using same process. These estimated values are used in histogram stretching with respect to Rayleigh distribution of R, G, and B components. As image is nonuniformly illuminated there are dark and bright patches in the image, so stretching is performed locally on small patches of image, instead of global stretching. A limit of $1 \%$ is applied to all three color components at minimum and maximum values. The limits are necessary to avoid underand oversaturation [15]. The stretching process is applied in the range of $1 \%$ to $99 \%$. Histogram stretching performed on each color channel with respect to Rayleigh distribution is given by the following:

$$
i_{\text {out }}=i_{\text {min }}+\left[2 * \alpha^{2} * \ln \left(\frac{1}{p_{i}(i)}\right)\right]^{1 / 2},
$$

where $i_{\text {out }}$ is pixel value in transformed image, $i_{\min }$ is minimum pixel value in the transformed image, $\alpha$ is parameter value, and $p_{i}(i)$ is cumulative distribution function of pixel values of input image. The effect of histogram stretching is shown in Figure 3. Figure 3 gives histogram of red, green, and blue components after and before stretching along with images.

\section{Image Quality Metrics}

The image quality metrics used in this paper are no-reference color image quality metrics. These metrics are based on primary and fundamental vision parameters perceived by human vision system, which are luminance, contrast, information content, and added noise. According to human vision perception a good quality image should have appropriate luminance, information content, and contrast. These four vision parameters can be measured using mathematical model given by Xie and Wang [16]. These are average contrast (AC), average information entropy (AIE), and average luminance $(\mathrm{AL})$. 
Images

\section{Original image 1}

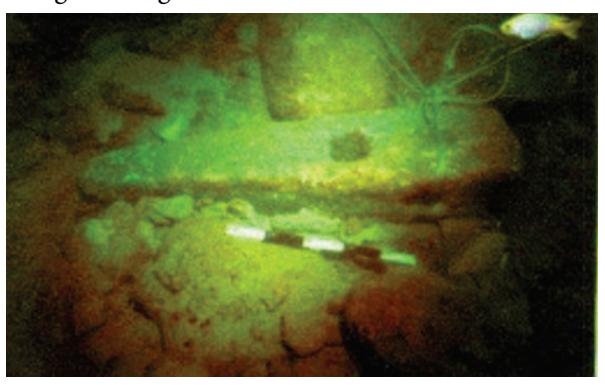

Processed image 1

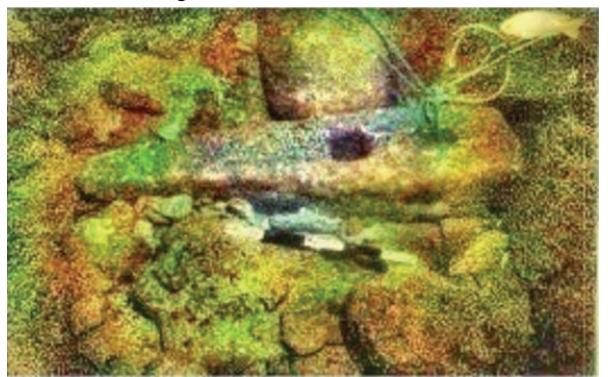

Original image 2

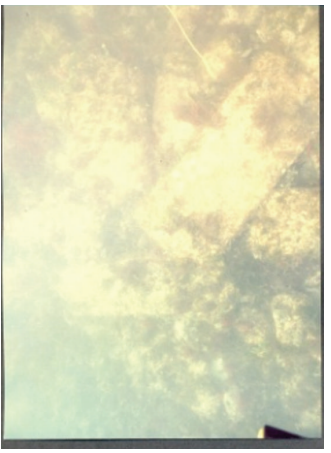

Processed image 2

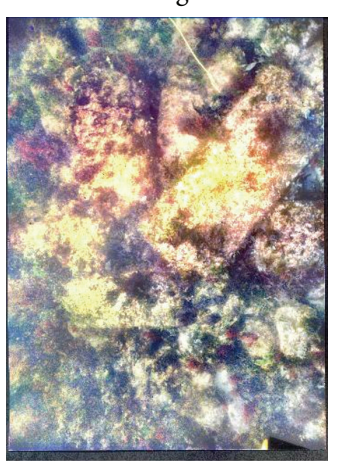

Histogram of red, green, and blue components

Red component Green component Blue component

of original image $1 \quad$ of original image $1 \quad$ of original image 1
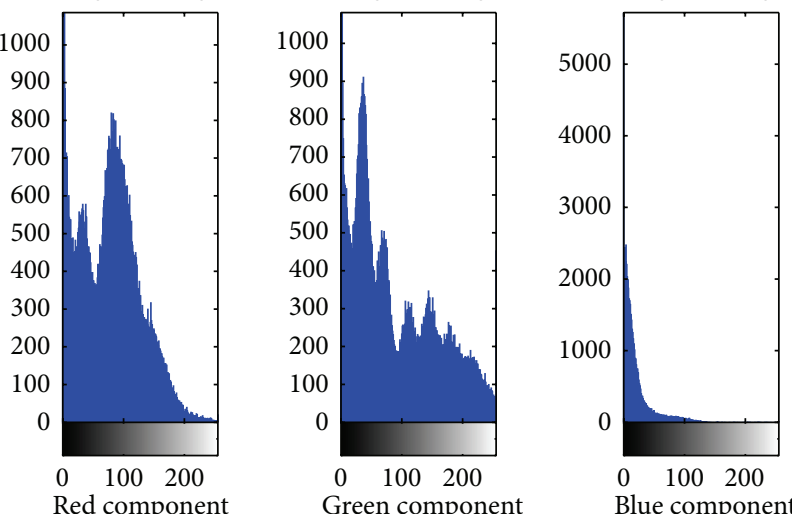

of processed image

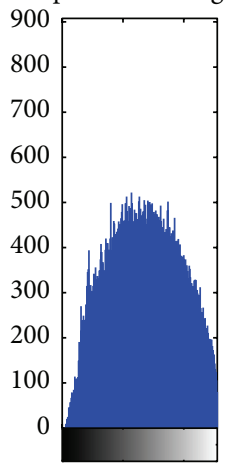

$\begin{array}{lll}0 & 100 & 200\end{array}$

Red component

of original image 2

$\times 10^{4}$

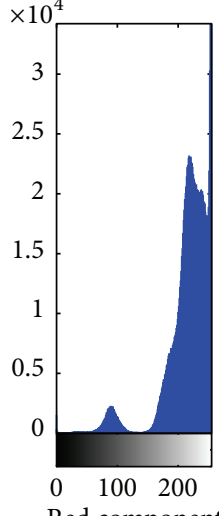

Red componen

of processed image 2

$\times 10^{4}$

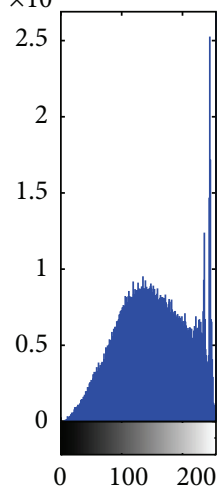

Green component

of processed image 1

Blue component

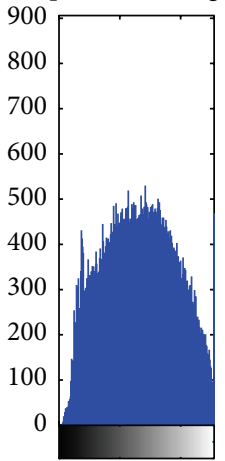

$\begin{array}{lll}0 & 100 & 200\end{array}$

Green component

of original image 2

$\times 10^{4}$

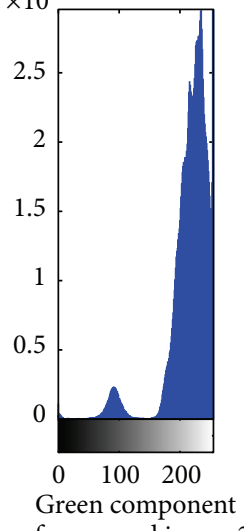

of processed image
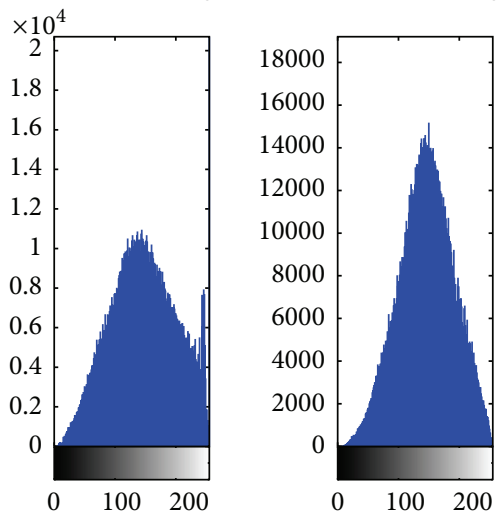

of processed image 1

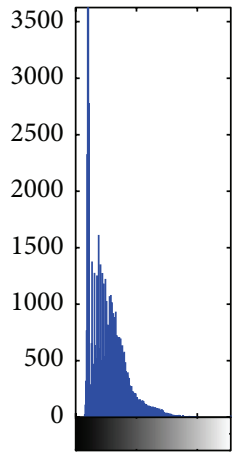

$$
0 \quad 100 \quad 200
$$

Blue component

of original image 2

$\times 10^{4}$

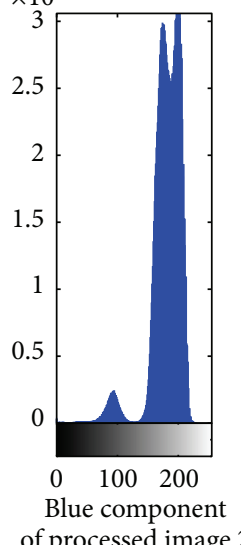

FIgURE 3: Continued. 
Images

Original image 3

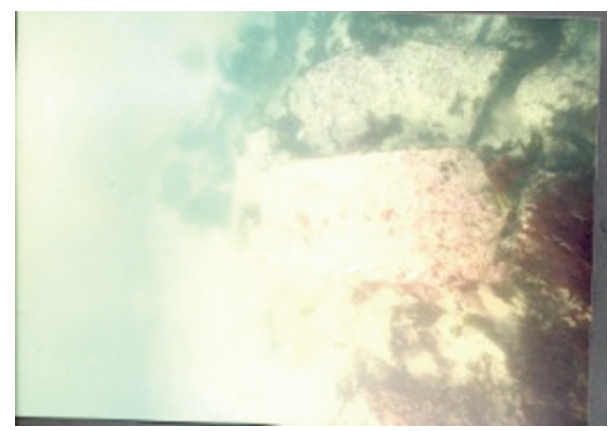

Processed image 3

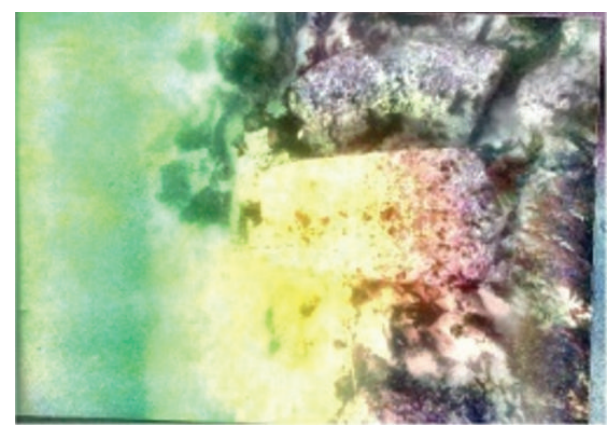

Original image 4

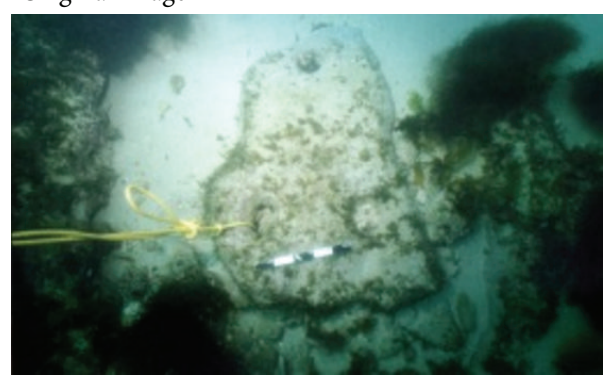

Processed image 4

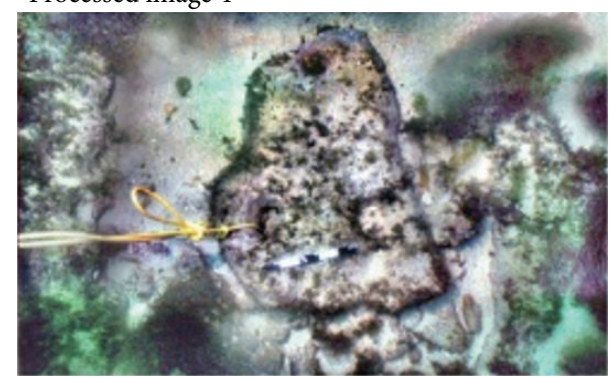

Histogram of red, green, and blue components

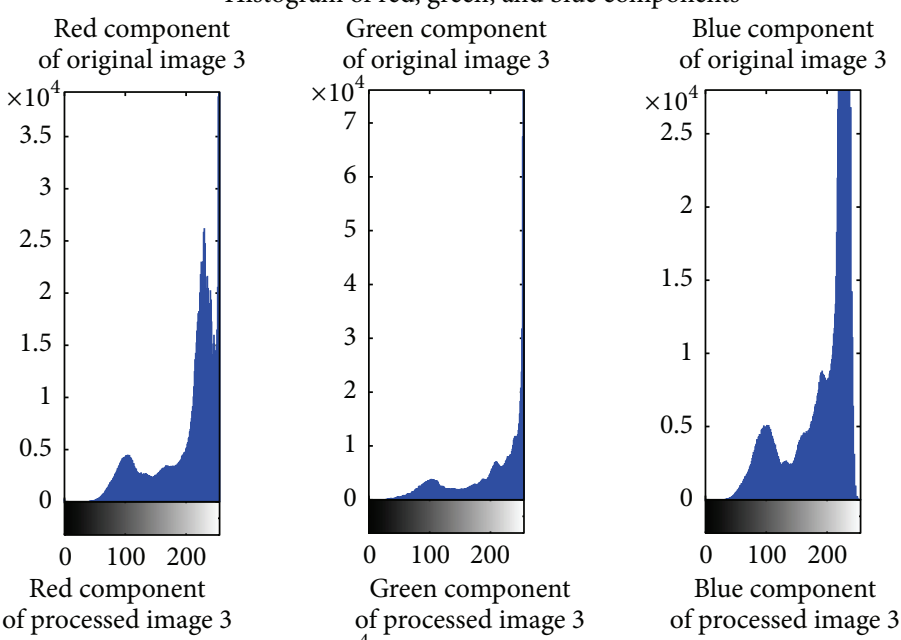

$\times 10^{4}$

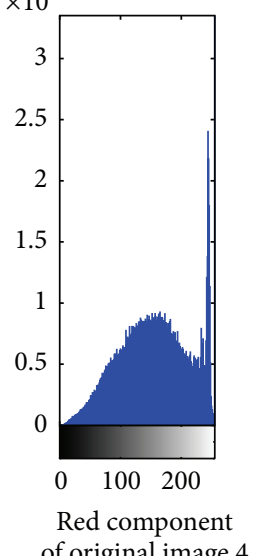
$\times 10^{4}$

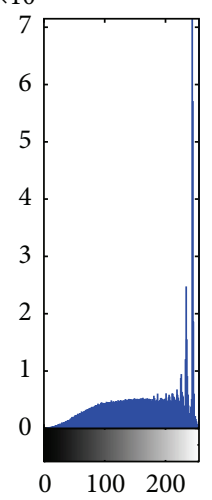

Green component of original image 4

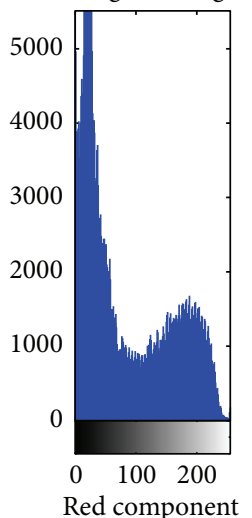

of processed image 4
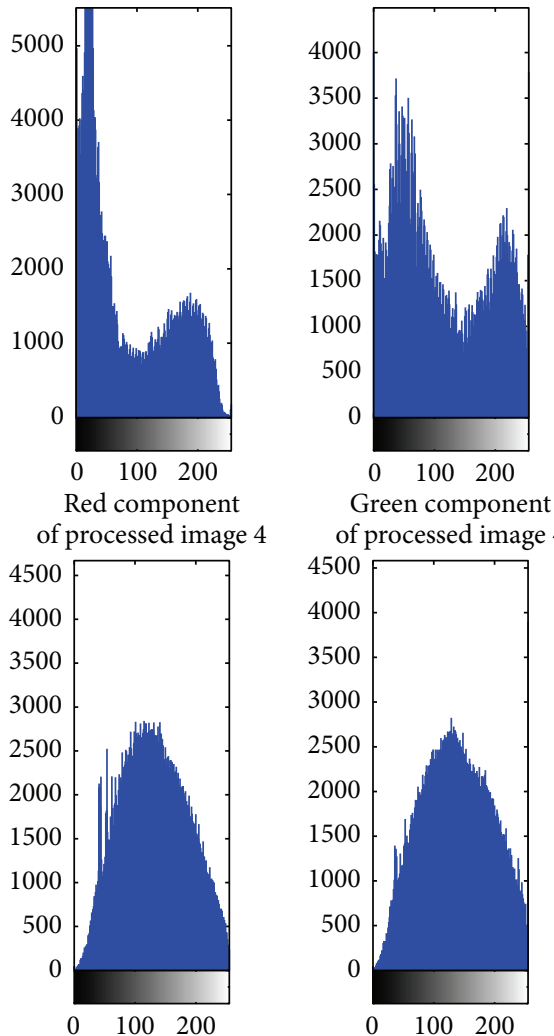

Green component of processed image 4

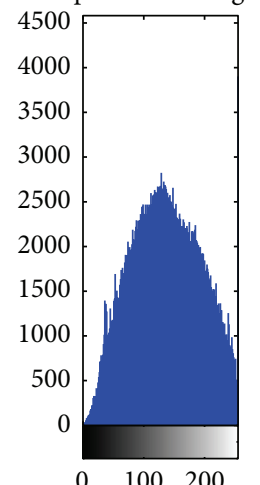

Blue component

of original image 4

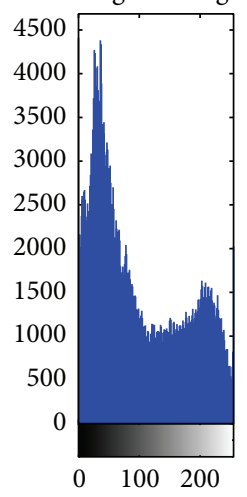

Blue component of processed image 4

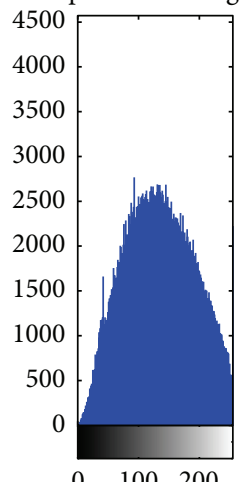

Figure 3: Continued. 
Images

Original image 5

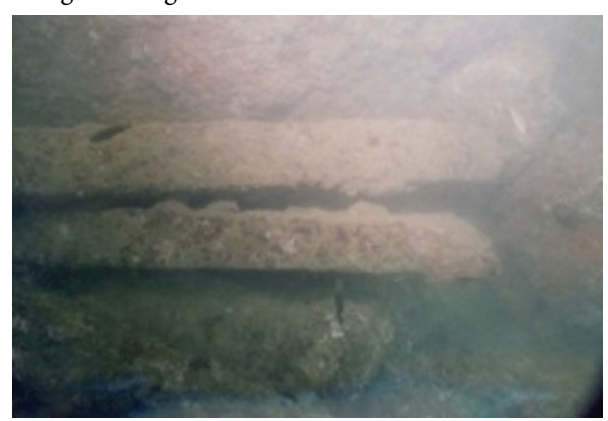

Processed image 5

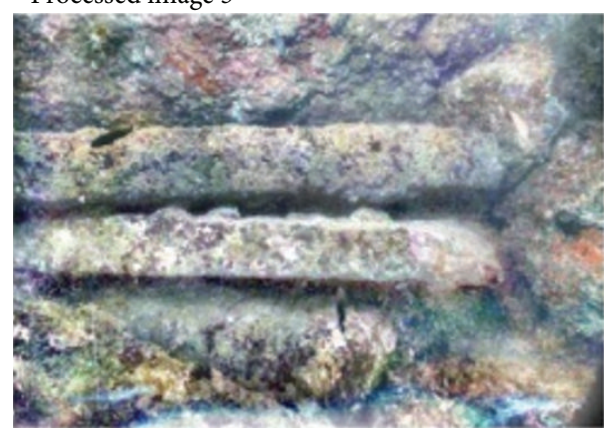

Histogram of red, green, and blue components

Red component Green component of original image 5
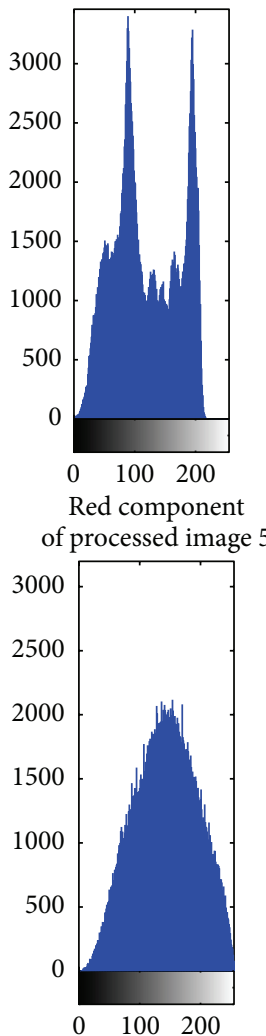

of original image 5

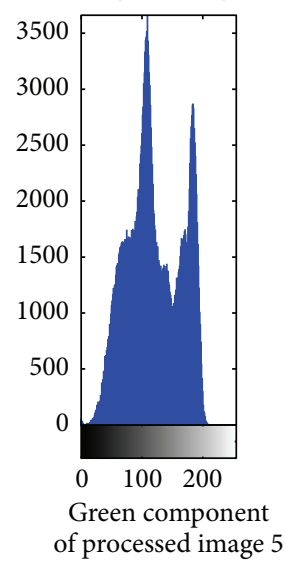

Blue component

of original image 5

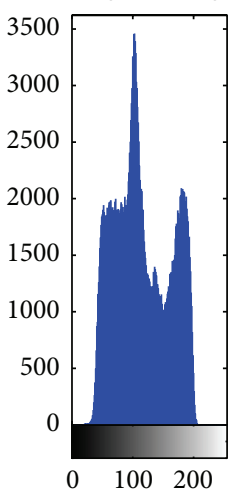

Blue component of processed image 5
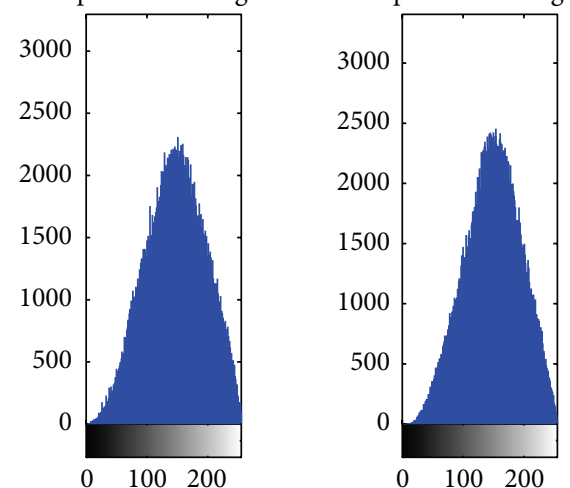

FIgURE 3: Original and processed images with their histogram for three color components (Original Image Courtesy NIO, Goa, India).

Average contrast is computed as

$$
\mathrm{AC}=\frac{1}{(M-1) \times(N-1)} \sum_{x=0}^{M-2} \sum_{y=0}^{N-2} C(x, y),
$$

where $C(x, y)$ is magnitude of mean of gradients of three color components $\mathrm{R}, \mathrm{G}$, and $\mathrm{B}$ given as

$$
C=\frac{1}{\sqrt{3}} \sqrt{\mathrm{GradR}^{2}+\mathrm{GradG}^{2}+\mathrm{GradB}^{2}} .
$$

Information entropy for single color channel is computed as

$$
\mathrm{IE}=-\sum_{i=0}^{255} p(i) \log _{2} p(i)
$$

where $p(i)$ is probability of $i$ th color level.

Then using (14) IE is calculated for red, green, and blue channels as IER, IEG, and IEB, respectively. Total AIE is defined as

$$
\mathrm{AIE}=\frac{1}{\sqrt{3}} \sqrt{\mathrm{IER}^{2}+\mathrm{IEG}^{2}+\mathrm{IEB}^{2}} .
$$

The maximal value of AIE for color image is 8 bits.
Average luminance is computed as follows:

$$
\mathrm{AL}=\frac{1}{M \times N} \sum_{x=0}^{M-1} \sum_{y=0}^{N-1} l(x, y) .
$$

$M$ and $N$ are rows and columns of the image and $l(x, y)$ is luminance value at pixel $(x, y)$. When histogram of luminance component $(l(x, y) \in[0,255])$ of an image is equalized then ideal value of AL should be 127.5.

So 127.5 is considered as an optimum value of luminance (OL) [16].

Comprehensive assessment function (CAF) is a general image quality assessment function given by Xie and Wang [16] computed as follows:

$$
\mathrm{CAF}=\mathrm{AIE}^{\alpha} \times \mathrm{AC}^{\beta} \times \mathrm{NNF}^{\gamma},
$$

where $\alpha, \beta$, and $\gamma$ are weight parameters; through experiment and comparison with subjective assessment Xie and Wang decided its values as $1,1 / 4$, and 3 , respectively [16]. In the above equation (17) AIE is average information entropy computed using (15), AC is average contrast computed using (12), and NNF is normalized neighborhood function defined as

$$
\mathrm{NNF}=\frac{\mathrm{OL}-\operatorname{dist}(\mathrm{AL}, \mathrm{OL})}{\mathrm{OL}}
$$



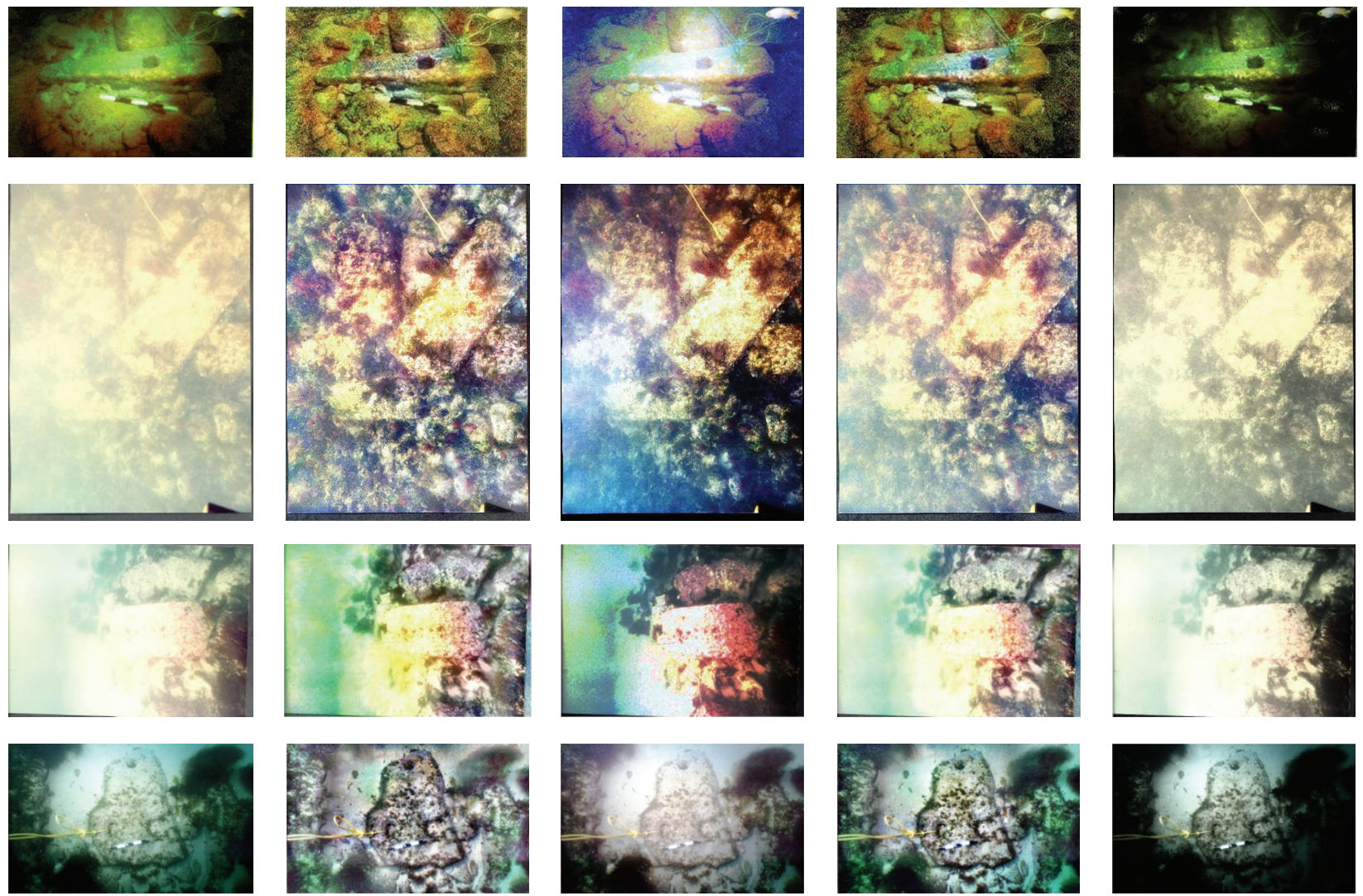

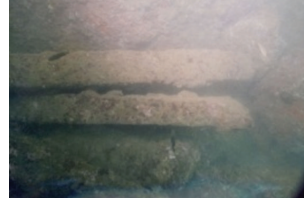

(a) Original image

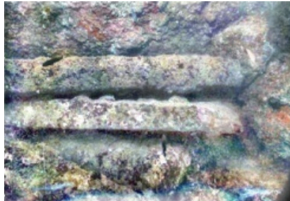

(b) Proposed method

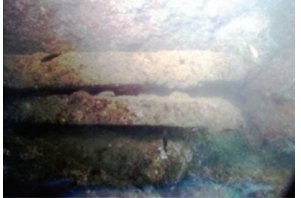

(c) Histogram equalization method

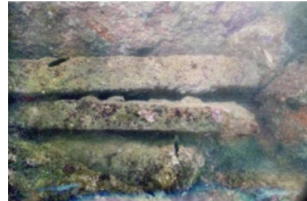

(d) Adaptive histogram equalization method

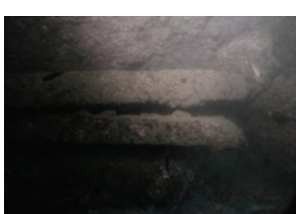

(e) Homomorphic filter method

FIGURE 4: Results of all four methods.

where OL is optimum luminance value and here it is considered [16] 127.5 and dist is absolute value. When AL is equal to OL then $\operatorname{dist}(\mathrm{AL}, \mathrm{OL})$ is zero and NNF has its optimum value of 1 . CAF computed by (17) is a convex function [16] and if the CAF is larger then the image quality is better.

\section{Results}

The proposed method is applied to five nonuniform illuminated images. The results are compared with the results of traditional methods of nonuniform illumination correction, which are histogram equalization $(\mathrm{HE})$, adaptive histogram equalization (AHE), and homomorphic filter.

Comparison of results is performed using no-reference image quality metrics for color images given in previous section.
The results are shown in Figure 4. Table 1 shows quantitative comparison of image quality metrics of original and processed images. The superior results are indicated in bold fonts in the table.

The parameters values for homomorphic filter are selected as given by Bazeille et al. [9].

\section{Conclusion}

The proposed method is used for nonuniform illumination correction of underwater images. Nonuniform illumination affects the overall contrast of the image. The proposed method shows improvement in all the quality metrics when compared with original image. Also there is improvement in quality parameters like average contrast, average information entropy, and comprehensive assessment function when the results are compared with results of traditional methods. 
TABLE 1: Comparison of results.

\begin{tabular}{|c|c|c|c|c|c|}
\hline Method & $\mathrm{AC}$ & AIE & $\mathrm{AL}$ & NNF & CAF \\
\hline \multicolumn{6}{|c|}{ Image 1} \\
\hline Original & 7.88 & 6.68 & 80.35 & 0.63 & 8.61 \\
\hline $\begin{array}{l}\text { Proposed } \\
\text { method }\end{array}$ & 22.93 & 7.11 & 121.38 & 0.95 & 9.70 \\
\hline $\mathrm{HE}$ & 16.13 & 5.32 & 127.78 & 0.99 & 8.32 \\
\hline AHE & 20.19 & 6.91 & 103.55 & 0.81 & 9.57 \\
\hline $\begin{array}{l}\text { Homomorphic } \\
\text { filter }\end{array}$ & 5.69 & 5.49 & 47.39 & 0.37 & 7.09 \\
\hline \multicolumn{6}{|c|}{ Image 2} \\
\hline Original & 7.3 & 6.37 & 199.46 & 0.44 & 7.08 \\
\hline $\begin{array}{l}\text { Proposed } \\
\text { method }\end{array}$ & 9.97 & 7.53 & 148.61 & 0.83 & 9.85 \\
\hline $\mathrm{HE}$ & 7.17 & 5.76 & 125.88 & 0.98 & 8.35 \\
\hline AHE & 6.31 & 7.23 & 167.6 & 0.68 & 9.14 \\
\hline $\begin{array}{l}\text { Homomorphic } \\
\text { filter }\end{array}$ & 3.53 & 7.61 & 176.86 & 0.61 & 8.61 \\
\hline \multicolumn{6}{|c|}{ Image 3} \\
\hline Original & 2.44 & 6.4 & 198 & 0.45 & 7.74 \\
\hline $\begin{array}{l}\text { Proposed } \\
\text { method }\end{array}$ & 9.1 & 7.04 & 171.11 & 0.66 & 9.10 \\
\hline $\mathrm{HE}$ & 9.92 & 5.25 & 130.69 & 0.97 & 7.96 \\
\hline AHE & 6.18 & 6.81 & 180.71 & 0.58 & 8.59 \\
\hline $\begin{array}{l}\text { Homomorphic } \\
\text { filter }\end{array}$ & 3.22 & 6.44 & 180.52 & 0.58 & 7.98 \\
\hline \multicolumn{6}{|c|}{ Image 4} \\
\hline Original & 5.03 & 7.75 & 104.38 & 0.81 & 9.8 \\
\hline $\begin{array}{l}\text { Proposed } \\
\text { method }\end{array}$ & 15.71 & 7.79 & 131.34 & 0.97 & 10.69 \\
\hline $\mathrm{HE}$ & 5.94 & 5.97 & 125.51 & 0.98 & 8.48 \\
\hline AHE & 12.00 & 7.84 & 115.34 & 0.9 & 10.4 \\
\hline $\begin{array}{l}\text { Homomorphic } \\
\text { filter }\end{array}$ & 4.09 & 6.7 & 68.64 & 0.53 & 8.29 \\
\hline \multicolumn{6}{|c|}{ Image 5} \\
\hline Original & 2.17 & 7.37 & 118.57 & 0.93 & 9.38 \\
\hline $\begin{array}{l}\text { Proposed } \\
\text { method }\end{array}$ & 11.67 & 7.61 & 141.23 & 0.89 & 10.18 \\
\hline $\mathrm{HE}$ & 6.85 & 7.45 & 124.49 & 0.97 & 9.99 \\
\hline AHE & 4.85 & 6.69 & 116.5 & 0.91 & 9.2 \\
\hline $\begin{array}{l}\text { Homomorphic } \\
\text { filter }\end{array}$ & 1.84 & 6.94 & 63.59 & 0.49 & 8.23 \\
\hline
\end{tabular}

But there is small degradation in average luminance and normalized neighborhood function compared to the results of traditional methods. However the degradation is by very small amount as compared to the improvements in other quality parameters. Also general image quality assessment function (CAF) which includes all other quality parameters (AC, AIE, and NNF) shows improvement for proposed method when compared with other methods. So the proposed method shows improvement in the results.

\section{Conflict of Interests}

The authors declare that there is no conflict of interests regarding the publication of this paper.

\section{References}

[1] J. S. Jaffe, "Computer modeling and the design of optimal underwater imaging systems," IEEE Journal of Oceanic Engineering, vol. 15, no. 2, pp. 101-111, 1990.

[2] R. Schettini and S. Corchs, "Underwater image processing: state of the art of restoration and image enhancement methods," EURASIP Journal on Advances in Signal Processing, vol. 2010, Article ID 746052, 2010.

[3] P. N. Andono, I. K. E. Purnama, and M. Hariad, "Underwater image enhancementusing adaptive filteringng for enhanced sift-based image matching," Journal of Theoretical and Applied Information Technology, vol. 51, no. 3, pp. 392-399, 2013.

[4] A. S. Abdul Ghani and N. A. M. Isa, "Underwater image quality enhancement through composition of dual-intensity images and Rayleigh-stretching," SpringerPlus, vol. 3, article 757, 2014.

[5] M. S. Hitam, W. N. J. H. W. Yussof, E. A. Awalludin, and Z. Bachok, "Mixture contrast limited adaptive histogram equalization for underwater image enhancement," in Proceedings of the IEEE International Conference on Computer Applications Technology (ICCAT '13), pp. 1-5, IEEE, Sousse, Tunisia, January 2013.

[6] R. Eustice, O. Pizarro, H. Singh, and J. Howland, "UWIT: underwater image toolbox for optical image processing and mosaicking in MATLAB," in Proceedings of the International Symposium on Underwater Technology, pp. 141-145, Tokyo, Japan, April 2002.

[7] A. Papoulis and S. Unnikrishna Pillai, Probability, Random Variables and Stochastic Processes, chapter 4\&8, Tata McGrawHill Publishing Company Limited, New Delhi, India, 2007.

[8] A. Arnold-Bos, J.-P. Malkasset, and G. Kervern, "Towards a model-free denoising of underwater optical images," in Proceedings of the IEEE Europe Oceans Conference, vol. 1, pp. 527-532, Brest, France, June 2005.

[9] S. Bazeille, I. Quidu, L. Jaulin, and J. P. Malkasse, "Automatic underwater image pre-processing," in Caracterisation du Milieu Marin (CMM '06), Brest, France, October 2006.

[10] R. Garcia, T. Nicosevici, and X. Cufí, "On the way to solve lighting problems in underwater imaging," in Proceedings of the IEEE Oceans Conference Record, vol. 2, pp. 1018-1024, IEEE, October 2002.

[11] M. Borgetto, V. Rigaud, and J.-F. Lots, "Lighting correction for underwater mosaicking enhancement," in Proceedings of the 16th International Conference on Vision Interface, Halifax, Canada, 2003.

[12] C. J. Prabhakar and P. U. Praveen Kumar, "An image based technique for enhancement of underwater images," International Journal of Machine Intelligence, vol. 3, no. 4, pp. 217-224, 2011.

[13] K. Iqbal, R. A. Salam, A. Osman, and A. Z. Talib, "Underwater image enhancement using integrated color model," IAENG International Journal of Computer Science, vol. 34, article 2, 2007.

[14] K. Iqbal, M. Odetayo, A. James, R. A. Salam, and A. Z. H. Talib, "Enhancing the low quality images using unsupervised colour correction method," in Proceedings of the IEEE International Conference on Systems Man and Cybernetics (SMC '10), pp. 17031709, IEEE, Istanbul, Turkey, October 2010. 
[15] A. S. Abdul Ghani and N. A. M. Isa, "Underwater image quality enhancement through Rayleigh-stretching and averaging image planes," International Journal of Naval Architecture and Ocean Engineering, vol. 6, no. 4, pp. 840-866, 2014.

[16] Z.-X. Xie and Z. F. Wang, "Color image quality assessment based on image quality parameters perceived by human vision system," in Proceedings of the IEEE International Conference on Multimedia Technology (ICMT '10), pp. 1-4, Ningbo, China, October 2010. 


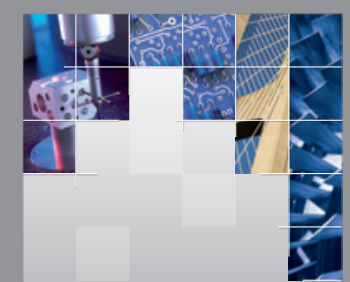

\section{Enfincering}
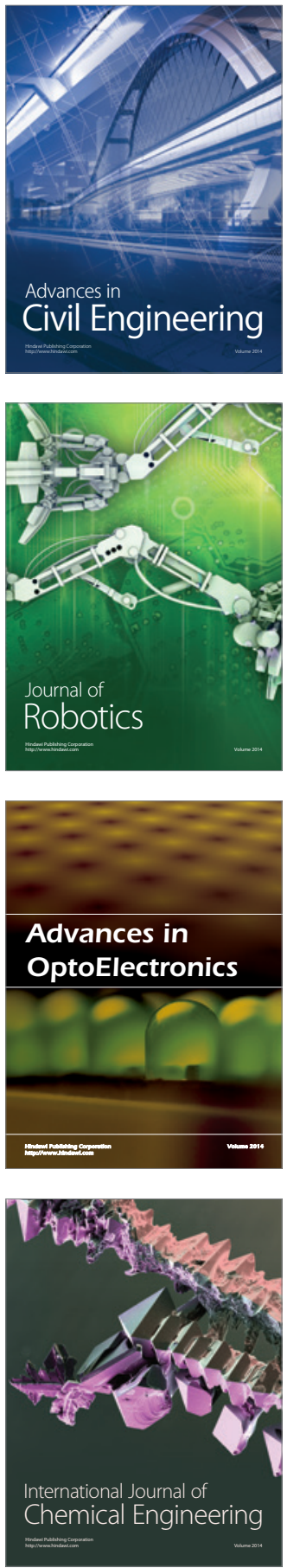

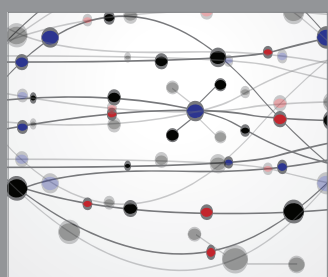

The Scientific World Journal

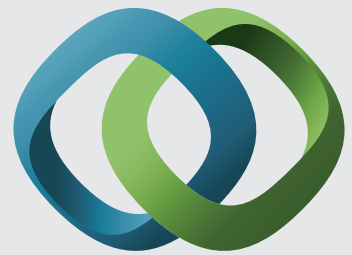

\section{Hindawi}

Submit your manuscripts at

http://www.hindawi.com
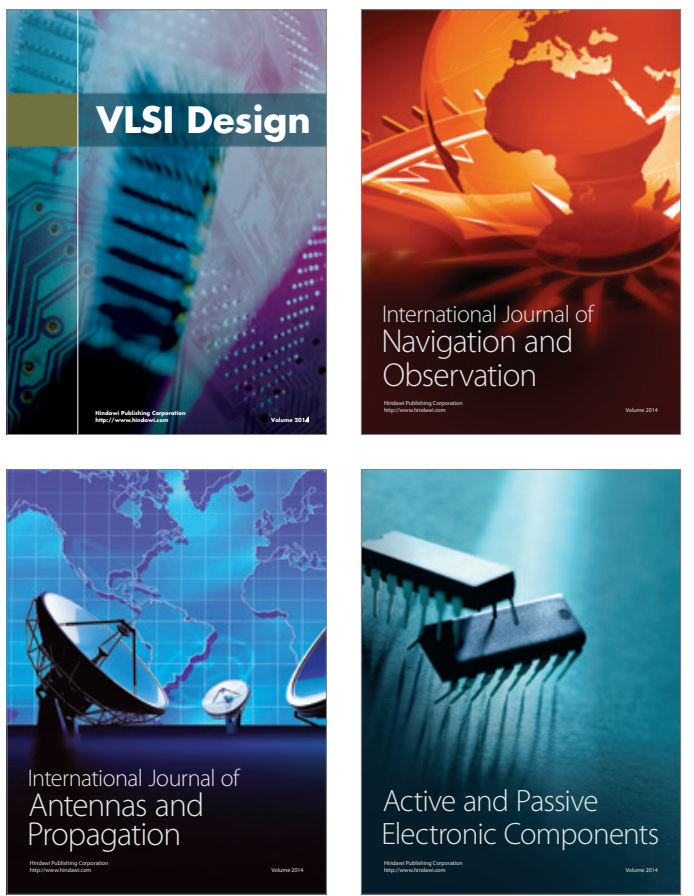
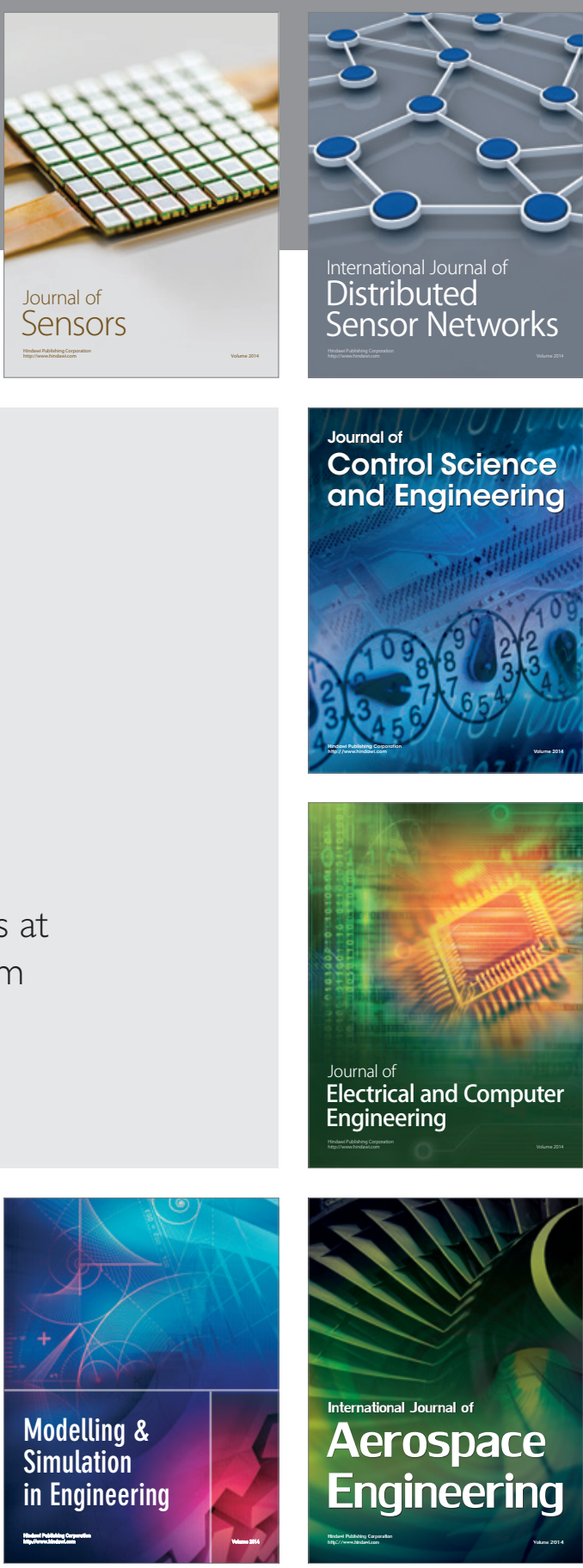

International Journal of

Distributed

Sensor Networks

Journal of

Control Science

and Engineering
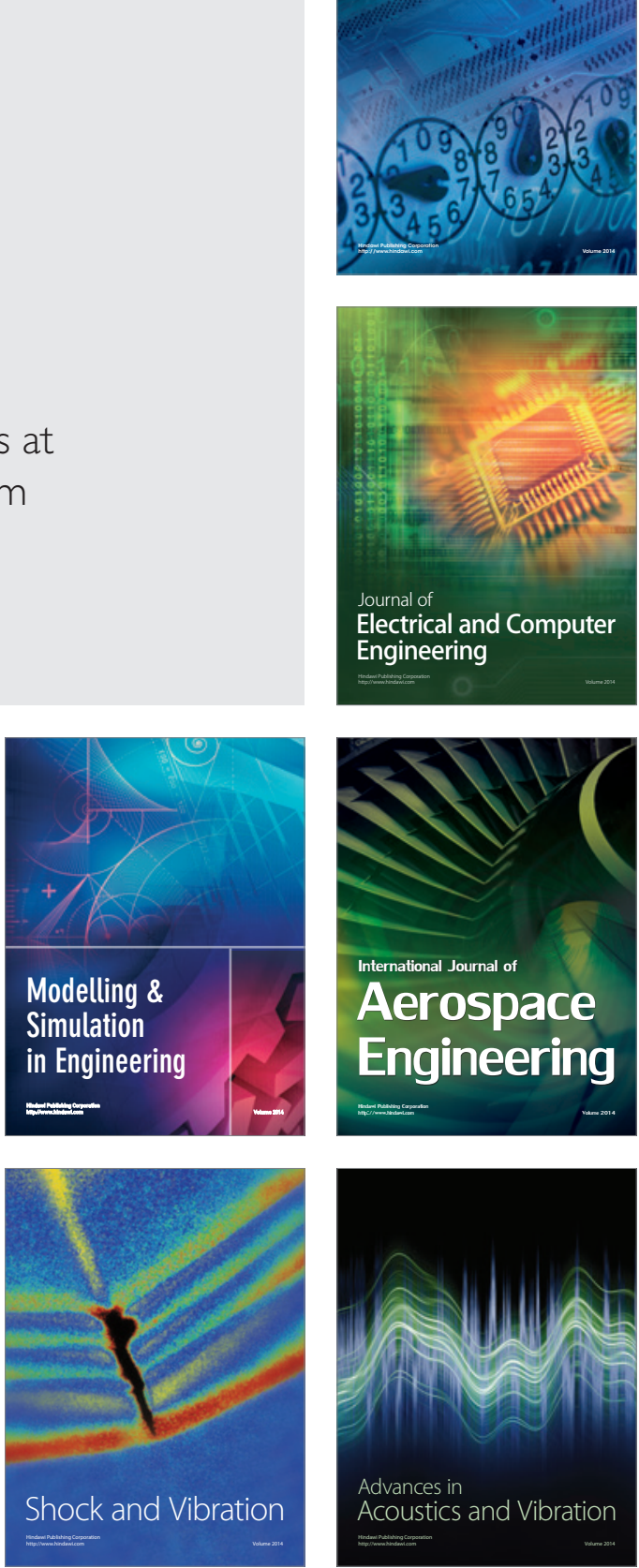